\title{
Translation groups of the boundary-layer flows induced by continuous moving surfaces
}

\section{Journal Article}

Author(s):

Magyari, Eugen

Publication date:

2010

Permanent link:

https://doi.org/10.3929/ethz-b-000157613

Rights / license:

In Copyright - Non-Commercial Use Permitted

Originally published in:

Journal of Fluid Mechanics 655, https://doi.org/10.1017/S0022112010000911 


\title{
Translation groups of the boundary-layer flows induced by continuous moving surfaces
}

\author{
EUGEN MAGYARI $\dagger$ \\ Institut für Hochbautechnik, ETH Zürich, Wolfgang-Pauli Strasse 1, CH-8093 Zürich, Switzerland
}

(Received 4 September 2009; revised 11 February 2010; accepted 11 February 2010;

first published online 27 May 2010)

The steady plane boundary-layer flows of velocity field $\{u(x, y), v(x, y)\}$ induced by continuous moving surfaces are revisited in this paper. It is shown that the governing balance equations, as well as the asymptotic condition $u(x, \infty)=0$ at the outer edge of the boundary layer are invariant under arbitrary translations $y \rightarrow y+y_{0}(x)$ of the transverse coordinate $y$. The wall conditions, i.e. the prescribed stretching velocity $u(x, 0) \equiv U_{w}(x)$ and the transpiration velocity $v(x, 0) \equiv V_{w}(x)$ distributions, however, undergo in general substantial changes. The consequences of this basic symmetry property on the structure of the solution space are investigated. It is found that starting with a primary solution which describes the boundary-layer flow induced by an impermeable surface, infinitely many translated solutions can be generated which form a continuous group, the translation group of the given primary solution. The elements of this group describe boundary-layer flows induced by permeable surfaces stretching under transformed wall conditions, $U_{w}(x) \rightarrow \tilde{U}_{w}(x)=u\left[x, y_{0}(x)\right]$ and $V_{w}(x) \rightarrow \tilde{V}_{w}(x)=v\left[x, y_{0}(x)\right]-y_{0}^{\prime}(x) u\left[x, y_{0}(x)\right]$, respectively. In this way, starting with a known solution $\{u(x, y), v(x, y)\}$ so that the inverse $y_{0}(x)=u^{-1}\left(x, \tilde{U}_{w}\right)$ of $u\left[x, y_{0}(x)\right]$ exists, a new solution $\{\tilde{u}(x, y), \tilde{v}(x, y)\}$ corresponding to any desired stretching velocity distribution $\tilde{U}_{w}(x)$ can be prepared. It also turns out that several exact solutions discovered during the latter decades are not basically new solutions, but translated counterparts of some formerly reported primary solutions. A few specific examples are discussed in detail.

\section{Introduction}

Flows driven by moving boundaries belong to the classical issues of fluid mechanics. Stokes' celebrated first and second problem, are the oldest examples of such flows. The well-known Couette flow (induced in a parallel plane channel by a moving boundary) also belongs to this class of phenomena. In addition to these classical cases, in some modern mechanical manufacturing and forming processes, in the stagnant ambient fluid a further important class of wall-driven flows with boundary-layer aspects arises. Examples of such processes are the drawing of plastic sheets by extrusion of a molten material through a narrow slot (Fisher 1976; Tadmor \& Klein 1970), the glass fibre and paper production, the melt spinning, the cooling of large metallic plates in a bath (Altan, Oh \& Gegel 1979), etc. The first investigation of the steady regimes of this kind of boundary-layer flows induced by continuous surfaces stretching in a quiescent ambient fluid was conducted by Sakiadis (1961). On this reason, in today's literature this type of boundary-layer flow is often referred to as 'Sakiadis flow'.

$\dagger$ Email address for correspondence: magyari@bluewin.ch 
Actually, a Sakiadis flow can be viewed as a Couette flow induced by a continuous surface moving in a semi-infinite space filled by a viscous fluid (the resting surface of the proper Couette set-up being shifted to infinity). Accordingly, the name CouetteSakiadis flow would reflect the historical development more appropriately.

Since the pioneering work of Sakiadis (1961) an enormous literature has been accumulated on this type of wall-driven flows. Following the early-stage papers of Ackroyd (1967), Tsou, Sparrow \& Goldstein (1967), Fox, Erickson \& Fan (1968), Crane (1970), Vleggaar (1977), Gupta \& Gupta (1977), Afzal \& Varshney (1980), Kuiken (1981) and of many other authors, the first comprehensive mathematical investigation of the steady self-similar Sakiadis flows has been reported by Banks (1983). Since then, the pertinent theory has considerably been extended and refined especially concerning the heat transfer features, the effect of a lateral mass flux as well as the unsteady flows (see e.g. Grubka \& Bobba 1985; Chen \& Char 1988; Ali 1995; Magyari \& Keller 1999, 2000; Magyari, Ali \& Keller 2001; Liao 2006; Liao $\&$ Magyari 2006). The main motivation for this research effort resides in the fact that features of the velocity and temperature boundary layers arising in the mentioned manufacturing processes exert a decisive influence on the quality of the final products. Indeed, during the cooling and solidifying of the processed molten material, the heat transferred through the boundary layer to the ambient fluid acts as a thermal treatment on the developing structure. In drawing of porous sheets by such technologies, this effect becomes additionally enhanced by the wall transpiration.

The formal analogy between the mathematics involved in the investigation of the Sakiadis flows on the one hand and in the investigation of some free-convection boundary-layer flows in fluid saturated porous media on the other hand, has brought, in spite of the basically different physics, a significant mutual fertilization for both of these two research fields. Some examples in this respect are the applicability of the Merkin transformation method (Merkin 1984; Magyari \& Keller 2005) in both of these areas, as well as the more recently discovered entrainment theorem (Magyari 2008; Magyari \& Rees 2008). For further aspects see also Cheng \& Minkowycz (1977), Ingham \& Brown (1986), Magyari \& Keller 2001; Pop \& Ingham (2001), Magyari, Pop \& Keller (2002a,b and 2003) and Magyari \& Rees (2006). This mathematical analogy is an additional reason why a comprehensive overview of the large number of solutions reported in the literature of the latter few decades is a fairly laborious task.

A screening of the pertinent literature emphasizes that especially with respect to the flows induced by impermeable and permeable moving surfaces, two parallel, seemingly independent research areas aroused. This insight is in fact one of the main motivations of the present paper which shows that owing to a basic symmetry property of the governing boundary-layer equations, namely their translation invariance, the high diversity of the known solutions can be reduced to a few generating solutions and, at the same time, new solutions can be generated by a systematic procedure. This issue is discussed in the paper in detail. Several specific examples which illustrate the consequences of translation invariance are presented.

\section{Basic equations}

The steady boundary-layer flow induced by a continuous surface stretching with velocity $U_{w}(x)$ in a quiescent incompressible fluid is governed by equations

$$
\left.\begin{array}{l}
u_{x}+v_{y}=0, \\
u u_{x}+v u_{y}=v u_{y y}
\end{array}\right\}
$$


subject to the boundary conditions

$$
u(x, 0)=U_{w}(x), \quad v(x, 0)=V_{w}(x), \quad u(x, \infty)=0 .
$$

The positive $x$-axis points in the direction of motion of the surface issuing from the slot (z-axis). The $y$-axis is perpendicular to $x$ and to the direction of the slot. $u$ and $v$ are the $x$ and $y$ components of the velocity field, respectively, $V_{w}(x)$ denotes the transpiration velocity distribution and $v$ stands for the kinematic viscosity of the fluid. The subscripts $x$ and $y$ denote partial derivatives with respect to these variables. In terms of the stream function $\psi=\psi(x, y)$ defined by equations $u=\psi_{y}, v=-\psi_{x}$, (2.1) and the boundary conditions (2.2) reduce to

$$
\psi_{y}(x, y) \psi_{x y}(x, y)-\psi_{x}(x, y) \psi_{y y}(x, y)=v \psi_{y y y}(x, y)
$$

and

respectively.

$$
\psi_{y}(x, 0)=U_{w}(x), \quad \psi_{x}(x, 0)=-V_{w}(x), \quad \psi_{y}(x, \infty)=0,
$$

\section{The translation invariance}

Since the boundary-layer equation (2.3) does not depend on the transverse coordinate $y$ explicitly, it is invariant under any uniform translation $y \rightarrow y+y_{0}$ of this coordinate. Moreover, owing to the basic assumption of Prandtl's boundarylayer theory that the thickness of the boundary layer is much smaller than the radius of curvature of the adjacent solid surface, in the governing momentum equation the curvature terms have been neglected. As a consequence, the $x$-momentum equation (2.3) must be invariant not only with respect to a uniform, but also with respect to any non-uniform translation

$$
y \rightarrow y+y_{0}(x)
$$

of the transverse coordinate $y$. The truth of this statement can be demonstrated easily by the direct transformation

$$
\psi(x, y) \rightarrow \psi\left[x, y+y_{0}(x)\right] \equiv \tilde{\psi}(x, y)
$$

of the stream function in (2.3) according to (3.1). Indeed, the transformation (3.1) leaves the $y$-derivatives of $\psi\left[x, y+y_{0}(x)\right]$ unchanged, while in the $x$-derivatives of $\psi\left[x, y+y_{0}(x)\right]$ besides $\psi_{x}\left[x, y+y_{0}(x)\right]$ the additional term $y_{0}^{\prime}(x) \psi_{y}\left[x, y+y_{0}(x)\right]$ occurs (everywhere in this paper the prime denotes differentiation with respect to the argument). Thus, the right-hand side of (2.3) is still $v \tilde{\psi}_{y y y}(x, y)$, while its left-hand side is transformed into

$$
\begin{gathered}
\psi_{y}\left[x, y+y_{0}(x)\right]\left\{\psi_{x y}\left[x, y+y_{0}(x)\right]+y_{0}^{\prime}(x) \psi_{y y}\left[x, y+y_{0}(x)\right]\right\} \\
-\left\{\psi_{x}\left[x, y+y_{0}(x)\right]+y_{0}^{\prime}(x) \psi_{y}\left[x, y+y_{0}(x)\right]\right\} \psi_{y y}\left[x, y+y_{0}(x)\right] .
\end{gathered}
$$

Now the terms $\pm y_{0}^{\prime}(x) \psi_{y}\left[x, y+y_{0}(x)\right] \psi_{y y}\left[x, y+y_{0}(x)\right]$ in the above expression cancel each other so that (2.3) is transformed by the translation (3.1) into

$$
\tilde{\psi}_{y}(x, y) \tilde{\psi}_{x y}(x, y)-\tilde{\psi}_{x}(x, y) \tilde{\psi}_{y y}(x, y)=v \tilde{\psi}_{y y y}(x, y) .
$$

In this way it is proven that when $\psi(x, y)$ is a solution of $(2.3)$, then $\tilde{\psi}(x, y)=$ $\psi\left[x, y+y_{0}(x)\right]$ also represents a solution of this equation. Consequently, the velocity components $u=\psi_{y}$ and $v=-\psi_{x}$ are transformed by (3.1) and (3.2) as follows:

$$
\left.\begin{array}{l}
u(x, y) \rightarrow \tilde{u}(x, y)=u\left[x, y+y_{0}(x)\right], \\
v(x, y) \rightarrow \tilde{v}(x, y)=v\left[x, y+y_{0}(x)\right]-y_{0}^{\prime}(x) \tilde{u}(x, y) .
\end{array}\right\}
$$


Accordingly, the boundary conditions (2.4) become

$$
\left.\begin{array}{l}
\tilde{u}(x, 0) \equiv \tilde{U}_{w}(x)=u\left[x, y_{0}(x)\right], \\
\tilde{v}(x, 0) \equiv \tilde{V}_{w}(x)=v\left[x, y_{0}(x)\right]-y_{0}^{\prime}(x) \tilde{U}_{w}(x)
\end{array}\right\} \text { on } y=0
$$

and

$$
\tilde{u}(x, y)=0 \quad \text { as } \quad y \rightarrow \infty .
$$

Therefore, the translation (3.1) of the transverse coordinate leaves the governing boundary-layer equation (2.3) as well as the asymptotic condition $\psi_{y}(x, \infty)=0$ invariant, but it changes the wall conditions essentially for any nontrivial displacement function $y_{0}(x)$. Accordingly, with the aid of the translations (3.1) one can generate physically new solutions from any specified solution $\{u(x, y), v(x, y)\}$ of the problem (2.1)-(2.2). The new solutions $\{\tilde{u}(x, y), \tilde{v}(x, y)\}$ given by (3.4) are associated with the stretching and transpiration velocity distributions $\left\{\tilde{U}_{w}(x), \tilde{V}_{w}(x)\right\}$ which, according to (3.5) deviate in general from the initial distributions $\left\{U_{w}(x), V_{w}(x)\right\}$ substantially. The transformed transpiration velocity $\tilde{V}_{w}(x)$ can be expressed in terms of the transformed stretching velocity $\tilde{U}_{w}(x)$ as

$$
\tilde{V}_{w}(x)=v\left[x, u^{-1}\left(x, \tilde{U}_{w}\right)\right]-\tilde{U}_{w}(x) \frac{\mathrm{d}}{\mathrm{d} x}\left[u^{-1}\left(x, \tilde{U}_{w}\right)\right]
$$

where $u^{-1}\left(x, \tilde{U}_{w}\right)$ stands for the inverse of the first (3.5) with respect to $y_{0}(x)$.

The solution corresponding to a given stretching velocity $U_{w}(x)$ of an impermeable surface (i.e. to the case $V_{w}(x) \equiv 0$ in $(2 b)$ ) will be referred to hereafter as a 'primary solution' of the boundary value problem (2.1)-(2.2). In the subsequent sections of the paper, the effect of translation invariance of (2.3) on the primary solutions describing various steady boundary-layer flows will be illustrated by specific examples.

\section{The translation group}

The transformed stream functions $\tilde{\psi}(x, y)=\psi\left[x, y+y_{0}(x)\right]$ generated from a given solution $\psi=\psi(x, y)$ of (2.3) by displacements $y_{0}=y_{0}(x)$ of the transverse coordinate $y$ are the elements of a continuous group, the translation group. Indeed, there exists a binary operation (a composition rule) under which the elements of the set $\left\{\psi\left[x, y+y_{0}(x)\right]\right\}$ are transformed in each other. This operation is the addition of two successive displacements $y_{01}(x)$ and $y_{02}(x)$ to a resulting one, $y_{03}(x)=y_{01}(x)+$ $y_{02}(x)$. Thus, the composition rule is associative and commutative. The identity element corresponds to the zero translation, and the inverse element corresponds to the opposite displacement $-y_{0}(x)$. Consequently, the set of solutions $\{\tilde{\psi}(x, y)\}$ of (2.3) generated by the translations (3.1) from some given solution $\psi(x, y)$ forms a continuous group. This group will be referred to as the translation group of the solution $\psi(x, y)$. Our main interest in the present paper will be focused on the translation groups of primary solutions, i.e. of the solutions $\psi(x, y)$ corresponding to given stretching velocity distributions $U_{w}(x)$ of impermeable surfaces, $V_{w}(x) \equiv 0$.

\section{Translations of the self-similar solutions}

\subsection{The general translations}

As it has been shown long time ago by Goldstein (1939), the steady plane boundarylayer equations admit two basic types of self-similar solutions which are characterized either by a power law, or by an exponential dependence on the wall coordinate $x$. This 
feature holds also in the present case of the wall-driven flows, when the stretching velocity $U_{w}(x)$ is a power law, or an exponential function of $x$. For the sake of simplicity, we consider in the present paper only the case of power law similarity and write the stretching velocity in the form

$$
U_{w}(x)=a x^{m},
$$

where $a$ is a dimensional scale factor which specifies the stretching velocity at the unit distance $x=1 \mathrm{~m}$ from the slot, $a=U_{w}(1)$. Basically, the dimensionless exponent $m$ may take in (5.1) any value between $\pm \infty$. As it is well known, the components of the velocity field can be written in this case in the form

$$
\left.\begin{array}{l}
u(x, y)=U_{w}(x) f^{\prime}(\eta), \quad \eta=\sqrt{\frac{\left|(m+1) U_{w}(x)\right|}{2 v x}} y, \\
v(x, y)=-s \sqrt{\frac{v\left|(m+1) U_{w}(x)\right|}{2 x}}\left[f(\eta)+\frac{m-1}{m+1} \eta f^{\prime}(\eta)\right],
\end{array}\right\}
$$

where the dimensionless stream function $f=f(\eta)$ is obtained as solution of the two point boundary value problem

$$
\begin{gathered}
s f^{\prime \prime \prime}+f f^{\prime \prime}-\frac{2 m}{m+1} f^{\prime 2}=0, \\
f(0)=f_{w}, \quad f^{\prime}(0)=1, \quad f^{\prime}(\infty)=0 .
\end{gathered}
$$

Here $f_{w}$ denotes the transpiration (or mass transfer) parameter and $s$ stands for the sign

$$
s=\operatorname{sgn}(a) \operatorname{sgn}(m+1) .
$$

The dimensional transpiration velocity $v(x, 0)=V_{w}(x)$ and the dimensional entrainment velocity $v(x, \infty)=V_{\infty}(x)$ are

$$
V_{w}(x)=-s \sqrt{\frac{v\left|(m+1) U_{w}(x)\right|}{2 x}} f_{w}, \quad V_{\infty}(x)=-s \sqrt{\frac{v\left|(m+1) U_{w}(x)\right|}{2 x}} f_{\infty},
$$

where $f_{\infty} \equiv f(\infty)$ denotes the dimensionless entrainment velocity. The primary solutions are obtained now from (5.2) to (5.6) for $f_{w}=0$. As shown by Magyari et al. (2002b), the case of inverse linear stretching velocity $U_{w}(x)=a / x$ corresponding to $m=-1$, requires a special approach and will not further be considered here. We throughout assume in this paper that $m \neq-1$.

The scale factor $a$ of the stretching velocity (5.1) is positive when the surface issues from the slot and moves toward $+\infty$, and negative when the surface moves from $+\infty$ toward $x=0$, where it enters the slot. In the first case $\left(U_{w}>0\right)$ one speaks usually about a stretching and in the second case $\left(U_{w}<0\right)$ about a shrinking surface. The most important point, however, is that these motions induce in the fluid two basically different velocity boundary layers, which, according to the nomenclature introduced by Goldstein (1965), are called forward (or usual) and backward boundary layers, respectively. The main physical difference between these two types of boundary layers originates from the fact that, in the case $U_{w}>0$ the slot plays the role of a definite leading edge, while in the case $U_{w}<0$, the leading edge recedes to an indefinite station far upstream. As a consequence, in the backward boundary layer (on a shrinking surface) the fluid loses any memory of the perturbation introduced by the indefinite leading edge. In this case, the slot at $x=0$ plays the role of a trailing edge. This is the reason why the forward and backward boundary-layer flows 
represent quite distinct physical phenomena. From mathematical point of view, the main difference occurs in the asymptotic decay of these two types of velocity boundary layers. Thus the forward boundary layers decay in general exponentially, while the backward ones decay algebraically as $y \rightarrow \infty$. However, as pointed out recently by Magyari \& Rees (2006) and by Liao \& Magyari (2006) some exponentially decaying boundary layers represent limiting cases of families of algebraically decaying ones. In the industrial manufacturing processes, both of these two types of boundary layers are of engineering interest. Forward boundary layers are generated in the ambient fluid, e.g. during the drawing of plastic sheets from a molten material, while, as reported by Kuiken (1981), during the cooling of a low-heat-resistance sheet, e.g. backward boundary layers may occur. is

According to (3.4), the transformed counterpart of the primary velocity field (5.2)

$$
\left.\begin{array}{rl}
\tilde{u}(x, y)= & U_{w}(x) f^{\prime}\left[\eta+\eta_{0}(x)\right], \\
\tilde{v}(x, y)= & -s \sqrt{\frac{v\left|(m+1) U_{w}(x)\right|}{2 x}}\left[f\left[\eta+\eta_{0}(x)\right]+\frac{m-1}{m+1}\right. \\
& \left.\times\left[\eta+\eta_{0}(x)\right] f^{\prime}\left[\eta+\eta_{0}(x)\right]\right]-y_{0}^{\prime}(x) \tilde{u}(x, y),
\end{array}\right\}
$$

where

$$
\eta_{0}(x)=\sqrt{\frac{\left|(m+1) U_{w}(x)\right|}{2 v x}} y_{0}(x)=\sqrt{\frac{|(m+1) a|}{2 v}} x^{(m-1) / 2} y_{0}(x)
$$

is the displacement of the similarity independent variable $\eta$ corresponding to the displacement $y_{0}(x)$ of the transverse coordinate $y$.

With the aid of (5.8), (5.7) can also be transcribed in the form

$$
\left.\begin{array}{rl}
\tilde{u}(x, y)= & U_{w}(x) f^{\prime}\left[\eta+\eta_{0}(x)\right], \\
\tilde{v}(x, y)= & -s \sqrt{\frac{v\left|(m+1) U_{w}(x)\right|}{2 x}}\left[f\left[\eta+\eta_{0}(x)\right]\right. \\
& \left.+\frac{(m-1) \eta+2 x \eta_{0}^{\prime}(x)}{m+1} f^{\prime}\left[\eta+\eta_{0}(x)\right]\right] .
\end{array}\right\}
$$

The stretching and transpiration velocity distributions which induce this transformed velocity field are

$$
\begin{aligned}
\tilde{U}_{w}(x) & =U_{w}(x) f^{\prime}\left[\eta_{0}(x)\right], \\
\tilde{V}_{w}(x) & =-s \sqrt{\frac{v\left|(m+1) U_{w}(x)\right|}{2 x}}\left[f\left[\eta_{0}(x)\right]+\frac{m-1}{m+1} \eta_{0}(x) f^{\prime}\left[\eta_{0}(x)\right]\right]-y_{0}^{\prime}(x) \tilde{U}_{w}(x) \\
& =-s \sqrt{\frac{v\left|(m+1) U_{w}(x)\right|}{2 x}}\left[f\left[\eta_{0}(x)\right]+\frac{2 x \eta_{0}^{\prime}(x)}{m+1} f^{\prime}\left[\eta_{0}(x)\right]\right] .
\end{aligned}
$$

Therefore, when the primary solution $f=f(\eta)$ of the original problem (5.3)-(5.4) is known and a displacement function $y_{0}(x)$ is specified, (5.7) or (5.9) give the corresponding transformed velocity field induced by the stretching and transpiration velocities (5.10). By this transformation, the self-similarity of the primary solution gets in general broken. When the function $f^{\prime}=f^{\prime}(\eta)$ admits an inverse, $f^{\prime-1}[\ldots]$, 
then the first (5.10) can be solved with respect to $\eta_{0}(x)$ explicitly,

$$
\eta_{0}(x)=f^{\prime-1}\left[\frac{\tilde{U}_{w}(x)}{U_{w}(x)}\right]
$$

This equation yields that special translation of the similarity independent variable $\eta$ which generates from a primary solution $\{u(x, y), v(x, y)\}$ precisely that new solution $\{\tilde{u}(x, y), \tilde{v}(x, y)\}$ which correspond to a desired stretching velocity distribution $\tilde{U}_{w}(x)$. Equations (6.4), (7.4) and (8.6) are specific examples for the inversion relationship (5.11).

Among the displacements (5.8) of the similarity variable $\eta$, the special case $\eta_{0}(x)=$ constant is of a basic physical interest since it preserves the self-similarity. The constant displacements of $\eta$ correspond to displacements $y_{0}(x)$ of the transverse coordinate $y$ which are proportional to $x^{-(m-1) / 2}$. Thus, the assumption $\eta_{0}(x)=$ constant requires in general non-uniform translations $y_{0}(x)$ of the coordinate $y$, except for the case $m=1$, where $y_{0}=\sqrt{v /|a|} \eta_{0}$ holds. The case $\eta_{0}(x)=$ constant will be considered in some detail below.

$$
\text { 5.2. The case } \eta_{0}(x)=\text { constant } \equiv \eta_{0}
$$

In this case the transformed velocity filed (5.9) reduces to

$$
\begin{aligned}
& \tilde{u}(x, y)=U_{w}(x) f^{\prime}\left(\eta+\eta_{0}\right), \\
& \tilde{v}(x, y)=-s \sqrt{\frac{v\left|(m+1) U_{w}(x)\right|}{2 x}}\left[f\left(\eta+\eta_{0}\right)+\frac{m-1}{m+1} \eta f^{\prime}\left(\eta+\eta_{0}\right)\right] .
\end{aligned}
$$

Accordingly, the transformed wall velocities (5.10) become

$$
\tilde{U}_{w}(x)=U_{w}(x) f^{\prime}\left(\eta_{0}\right), \tilde{V}_{w}(x)=-s \sqrt{\frac{v\left|(m+1) U_{w}(x)\right|}{2 x}} f\left(\eta_{0}\right) .
$$

The first (5.13) shows that for the special choice $\eta_{0}(x)=$ constant, the transformed stretching velocity $\tilde{U}_{w}(x)$ is always proportional to $U_{w}(x)$, in a full agreement with (5.11). According to (5.1), the constant of proportionality $f^{\prime}\left(\eta_{0}\right)$ can be absorbed in the scale factor $a$, so that in this case the transformed stretching velocity $\tilde{U}_{w}(x)$ is nothing more that a rescaled form of $U_{w}(x)$. At the same time, the second (5.13) shows that the transformed counterpart $\{\tilde{u}, \tilde{v}\}$ of the primary solution $\{u, v\}$ describes now the flow induced by a permeable surface with the transpiration velocity distribution $\tilde{V}_{w}(x)$. In the above sense, it is convenient to introduce the notations

$$
\tilde{a} \equiv a f^{\prime}\left(\eta_{0}\right), \quad \tilde{f}(\tilde{\eta}) \equiv \frac{f\left(\eta+\eta_{0}\right)}{\sqrt{f^{\prime}\left(\eta_{0}\right)}}, \quad \tilde{\eta} \equiv \sqrt{f^{\prime}\left(\eta_{0}\right)} \eta=\sqrt{\frac{\left|(m+1) \tilde{U}_{w}(x)\right|}{2 v x}} y,
$$

so that (5.13) become

$$
\tilde{U}_{w}(x)=\tilde{a} x^{m}, \quad \tilde{V}_{w}(x)=-s \sqrt{\frac{v\left|(m+1) \tilde{U}_{w}(x)\right|}{2 x}} \tilde{f}_{w},
$$

where

$$
\tilde{f}_{w} \equiv \tilde{f}(0)=\frac{f\left(\eta_{0}\right)}{\sqrt{f^{\prime}\left(\eta_{0}\right)}}
$$

is the transformed mass transfer parameter. The transformed dimensionless entrainment velocity $\tilde{f}_{\infty} \equiv \tilde{f}(\infty)$ is obtained as

$$
\tilde{f}_{\infty}=\frac{f_{\infty}}{\sqrt{f^{\prime}\left(\eta_{0}\right)}} .
$$


Furthermore, (5.14) imply

$$
\tilde{f}^{\prime}(\tilde{\eta})=\frac{f^{\prime}\left(\eta+\eta_{0}\right)}{f^{\prime}\left(\eta_{0}\right)},
$$

and thus (5.12) can be transcribed in the form

$$
\left.\begin{array}{l}
\tilde{u}(x, y)=\tilde{U}_{w}(x) \tilde{f}^{\prime}(\tilde{\eta}), \\
\tilde{v}(x, y)=-s \sqrt{\frac{v\left|(m+1) \tilde{U}_{w}(x)\right|}{2 x}}\left[\tilde{f}(\tilde{\eta})+\frac{m-1}{m+1} \tilde{\eta} \tilde{f}^{\prime}(\tilde{\eta})\right] \cdot
\end{array}\right\}
$$

Therefore, a translation with $\eta_{0}(x)=$ constant applied to a given primary solution $\left(f_{w}=0\right)$ generates a new solution which describes the flow corresponding to the physically equivalent power-law stretching velocity $\tilde{U}_{w}(x)=\tilde{a} x^{m}$ in the case when the surface is permeable and the transpiration velocity distribution $\tilde{V}_{w}(x)$ is effective. Equations (5.19) have the same form as (5.2). This comparison shows that the solutions associated with non-vanishing values of the mass transfer parameter $f_{w}$ do not represent basically new solutions of the boundary value problem (5.3)-(5.4), but are elements of the translation group of the primary solutions $\left(f_{w}=0\right)$ of this problem and, accordingly, can be generated from the latter ones by constant displacements of the similarity variable $\eta$. In this case the self-similarity is preserved. In order to be more specific, in $\S \S 6,7$ and 8 the translation groups of some exact analytical solutions of the boundary value problem (5.3)-(5.4) will be discussed in detail.

\subsection{The case $y_{0}(x)=$ constant $\equiv y_{0}$}

According to (5.8) the special case of constant displacements $y_{0}(x)=$ constant of the transverse coordinate is (for $m \neq 1$ ) a reciprocal one to the case of constant displacements $\eta_{0}(x)=$ constant of the similarity independent variable in the sense that, in contrast to the latter case which is realized when $y_{0}(x) \propto x^{-(m-1) / 2}$, the assumption $y_{0}(x)=$ constant implies $\eta_{0}(x) \propto x^{+(m-1) / 2}$. Nevertheless, the difference between these two cases is substantial, since for $y_{0}(x)=$ constant (with $m \neq 1$ ) in the corresponding transformed solution (5.7),

$\tilde{u}(x, y)=U_{w}(x) f^{\prime}\left[\eta+\eta_{0}(x)\right]$,

$\left.\tilde{v}(x, y)=-s \sqrt{\frac{v\left|(m+1) U_{w}(x)\right|}{2 x}}\left[f\left[\eta+\eta_{0}(x)\right]+\frac{m-1}{m+1}\left[\eta+\eta_{0}(x)\right] f^{\prime}\left[\eta+\eta_{0}(x)\right]\right],\right\}$

the self-similarity of the primary solution (5.2) gets broken. The stretching and transpiration velocity distributions which induce the velocity field (5.20) are

$$
\begin{aligned}
& \tilde{U}_{w}(x)=U_{w}(x) f^{\prime}\left[\eta_{0}(x)\right], \\
& \left.\tilde{V}_{w}(x)=-s \sqrt{\frac{v\left|(m+1) U_{w}(x)\right|}{2 x}}\left[f\left[\eta_{0}(x)\right]+\frac{m-1}{m+1} \eta_{0}(x) f^{\prime}\left[\eta_{0}(x)\right]\right] .\right\}
\end{aligned}
$$

\section{Translation group of the Crane solution}

The first exact analytical solution of the boundary value problem (2.1)-(2.2) has been reported by Crane (1970). Crane's self-similar solution

$$
\left.\begin{array}{rl}
f(\eta) & =1-\mathrm{e}^{-\eta}, \quad f^{\prime}(\eta)=\mathrm{e}^{-\eta}, \quad \eta=\sqrt{(a / v)} y \\
u(x, y) & =a x \mathrm{e}^{-\eta}, \quad v(x, y)=-\sqrt{a v}\left(1-\mathrm{e}^{-\eta}\right), \\
U_{w}(x) & =a x, \quad V_{w}(x)=0
\end{array}\right\}
$$


describes the flow induced by a continuous impermeable surface, $V_{w}(x)=0$, stretching with the linearly rising velocity $U_{w}(x)=a x$ and represents a forward velocity boundary layer of constant thickness $\delta=\sqrt{v / a}$ This is the special case $m=1, s=1$ of (5.2)-(5.6) with $a>0$. The dimensionless entrainment velocity for this primary solution is $f_{\infty} \equiv f(\infty)=1$.

\subsection{The general translation group of Crane's solution}

According to (5.9), the translation group of the Crane solution (6.1) is given by

$$
\left.\begin{array}{l}
\tilde{u}(x, y)=a x \mathrm{e}^{-\left[\eta+\eta_{0}(x)\right]}, \quad \eta_{0}(x)=\sqrt{(a / v)} y_{0}(x), \\
\tilde{v}(x, y)=-\sqrt{v a}\left[1-\mathrm{e}^{-\left[\eta+\eta_{0}(x)\right]}+\eta_{0}^{\prime}(x) x \mathrm{e}^{-\left[\eta+\eta_{0}(x)\right]}\right] .
\end{array}\right\}
$$

The transformed solution (6.2) corresponds to the wall functions

$$
\tilde{U}_{w}(x)=a x \mathrm{e}^{-\eta_{0}(x)}, \quad \tilde{V}_{w}(x)=-\sqrt{v a}\left[1-\mathrm{e}^{-\eta_{0}(x)}+\eta_{0}^{\prime}(x) x \mathrm{e}^{-\eta_{0}(x)}\right] .
$$

Equations (6.2) give in terms of the displacement function $\eta_{0}(x)=\sqrt{(a / v)} y_{0}(x)$ the translation group associated with the primary solution (6.1) in its most general form. In addition to this form, it is useful to express the transformed velocities $\tilde{u}(x, y)$ and $\tilde{v}(x, y)$ also in terms of the transformed stretching velocity $\tilde{U}_{w}(x)$, instead of $\eta_{0}(x)$. This can be achieved with the aid of (5.11) which in the present case becomes

$$
\eta_{0}(x)=-\ln \left[\frac{\tilde{U}_{w}(x)}{a x}\right] .
$$

Thus, expressed in terms of $\tilde{U}_{w}(x),(6.2) \mathrm{read}$

$$
\left.\begin{array}{l}
\tilde{u}(x, y)=\tilde{U}_{w}(x) \mathrm{e}^{-\eta}, \\
\tilde{v}(x, y)=-\sqrt{a v}\left[1-\frac{1}{a} \frac{\mathrm{d} \tilde{U}_{w}(x)}{\mathrm{d} x} \mathrm{e}^{-\eta}\right] \cdot
\end{array}\right\}
$$

The corresponding transpiration velocity distribution is obtained immediately as

$$
\tilde{V}_{w}(x)=-\sqrt{a v}\left[1-\frac{1}{a} \frac{\mathrm{d} \tilde{U}_{w}(x)}{\mathrm{d} x}\right] .
$$

This simple example emphasizes the astonishing consequences of the translation invariance clearly. Indeed, comparing the transformed solution (6.5) to the primary solution (6.1), the following features emerge.

(i) The same self-similar velocity boundary layer $\mathrm{e}^{-\eta}$ can be generated not only by the linear stretching velocity $U_{w}(x)=a x$ of an impermeable surface $\left(V_{w}(x)=0\right)$, but by any other arbitrary stretching velocity distribution $\tilde{U}_{w}(x)$ when the surface is permeable and a lateral suction/injection of the fluid with a suitable velocity distribution $\tilde{V}_{w}(x)$ is applied.

(ii) The suitable transpiration velocity $\tilde{V}_{w}(x)$ is obtained in terms of the stretching velocity $\tilde{U}_{w}(x)$ by the simple relationship (6.6). In other words, the change from the linear stretching velocity $U_{w}(x)=a x$ of an impermeable surface to an arbitrary stretching velocity distribution $\tilde{U}_{w}(x)$ of a permeable surface, can always be compensated by the effect of the transpiration velocity distribution (6.6), so that the similar velocity field $\mathrm{e}^{-\eta}$ remains unchanged, i.e.

$$
\mathrm{e}^{-\eta}=\frac{u(x, y)}{U_{w}(x)}=\frac{\tilde{u}(x, y)}{\tilde{U}_{w}(x)} .
$$

We see, therefore, that the similar velocity field $\mathrm{e}^{-\eta}$ is the same for the whole translation group of the primary solution (6.1), no matter the choice of $\tilde{U}_{w}(x)$. We also mention 
here that the generalized Crane solution given by (6.5) and (6.6) has also been deduced and illustrated by several specific examples recently by Weidman \& Magyari (2010) by a direct solution of the boundary value problem (2.1)-(2.2), without any reference to the translation invariance of the governing momentum equation which, as shown above, represents the deep physical reason for the existence of the solution (6.5). Accordingly, the generalized Crane solution (6.5) in fact is not a basically new solution of the boundary value problem (2.1)-(2.2), but it is a translated counterpart of the primary solution (6.1) of Crane.

\subsection{The case $\eta_{0}(x)=$ constant $\equiv \eta_{0}$}

Bearing in mind the primary solution $(6.1)$ for $f(\eta)$, the parameters $\tilde{f}_{w}, \tilde{f}_{\infty}$ and $\tilde{a}$ of the transformed solution obtained by a translation with $\eta_{0}(x)=$ constant, as well as the corresponding wall functions (5.15) become

$$
\begin{gathered}
\tilde{f}_{w}=\tilde{f}_{\infty}-\frac{1}{\tilde{f}_{\infty}}, \quad \tilde{f}_{\infty}=\mathrm{e}^{\eta_{0} / 2}, \quad \tilde{a}=a \mathrm{e}^{-\eta_{0}}=\frac{a}{\tilde{f}_{\infty}^{2}}, \\
\tilde{U}_{w}(x)=\tilde{a} x, \quad \tilde{V}_{w}(x)=-\sqrt{v \tilde{a}} \tilde{f}_{w} .
\end{gathered}
$$

The first (6.8) gives the value of the transformed entrainment velocity $\tilde{f}_{\infty}$ in terms of the transformed transpiration parameter $\tilde{f}_{w}$ as

$$
\tilde{f}_{\infty}=\frac{1}{2}\left(\tilde{f}_{w}+\sqrt{\tilde{f}_{w}^{2}+4}\right) .
$$

In this way, (5.14) and (5.19) yield for the transformed solution the expressions

$$
\tilde{u}(x, y)=\tilde{a} x \mathrm{e}^{-\tilde{f}_{\infty} \tilde{\eta}}, \quad \tilde{v}(x, y)=-\sqrt{\tilde{a} v}\left(\tilde{f}_{w}+\frac{1-\mathrm{e}^{-\tilde{f}_{\infty} \tilde{\eta}}}{\tilde{f}_{\infty}}\right), \quad \tilde{\eta}=\sqrt{\tilde{a} / v} y=\frac{\eta}{\tilde{f}_{\infty}} .
$$

In the transformed solution (6.11) one recovers the exact solution obtained by Gupta \& Gupta (1977) by a direct extension of Crane's result to the case of a permeable surface when the uniform transpiration velocity $\tilde{V}_{w}(x)=-\sqrt{v \tilde{a}} \tilde{f}_{w}$ is applied (see also Magyari \& Keller 2000). We see in this way clearly that (6.11) in fact is not a basically new solution of the boundary value problem, but it is the transformed counterpart with $\eta_{0}(x)=$ constant of the primary solution (6.1) of Crane. As mentioned above, for $m=1$ the cases $\eta_{0}(x)=$ constant and $y_{0}(x)=$ constant are equivalent since in this case the relationship $y_{0}=\sqrt{v /|a|} \eta_{0}$ holds.

\section{Translation group of the of the Bickley solution}

As it is well known, for $m=-1 / 3$ and $a>0$ the self-similar forward $(s=1)$ boundary-layer solution (5.2) can also be expressed in a closed analytical form (see e.g. Banks 1983). In the impermeable case $\left(f_{w}=0\right)$, this solution has the form

$$
\left.\begin{array}{l}
f(\eta)=\sqrt{2} \tanh \left(\frac{\eta}{\sqrt{2}}\right), \quad \eta=\sqrt{\frac{U_{w}(x)}{3 v x}} y, \quad U_{w}(x)=a x^{-1 / 3}, \\
u(x, y)=U_{w}(x) \operatorname{sech}^{2}\left(\frac{\eta}{\sqrt{2}}\right), \\
v(x, y)=-\sqrt{\frac{v U_{w}(x)}{3 x}}\left\{\sqrt{2} \tanh \left(\frac{\eta}{\sqrt{2}}\right)-2 \eta \operatorname{sech}^{2}\left(\frac{\eta}{\sqrt{2}}\right)\right\} .
\end{array}\right\}
$$


Since (7.1) coincide formally with the velocity field of the free plane jet which has first been reported by Bickley (1937), it will be referred to hereafter as the Bickley solution. The dimensionless entrainment velocity in this case is $f_{\infty} \equiv f(\infty)=\sqrt{2}$.

\subsection{The general translation group of Bickley's solution}

According to (5.9), the translation group of the primary solution (7.1) is given by

$$
\left.\begin{array}{l}
\tilde{u}(x, y)=U_{w}(x) \operatorname{sech}^{2}\left(\frac{\eta+\eta_{0}}{\sqrt{2}}\right), \quad \eta_{0}(x)=\sqrt{\frac{U_{w}(x)}{3 v x}} y_{0}(x), \\
\tilde{v}(x, y)=-\sqrt{\frac{v U_{w}(x)}{3 x}}\left\{\sqrt{2} \tanh \left(\frac{\eta+\eta_{0}}{\sqrt{2}}\right)-\left(2 \eta-3 x \eta_{0}^{\prime}\right) \operatorname{sech}^{2}\left(\frac{\eta+\eta_{0}}{\sqrt{2}}\right)\right\} .
\end{array}\right\}
$$

The stretching and the transpiration velocity distributions which induce this transformed field are

$$
\begin{aligned}
& \tilde{U}_{w}(x)=U_{w}(x) \operatorname{sech}^{2}\left(\frac{\eta_{0}}{\sqrt{2}}\right), \\
& \left.\tilde{V}_{w}(x)=-\sqrt{\frac{v U_{w}(x)}{3 x}}\left\{\sqrt{2} \tanh \left(\frac{\eta_{0}}{\sqrt{2}}\right)+3 x \eta_{0}^{\prime}(x) \operatorname{sech}^{2}\left(\frac{\eta_{0}}{\sqrt{2}}\right)\right\} \cdot\right\}
\end{aligned}
$$

Equations (7.2) give in terms of the displacement function $\eta_{0}(x)=\sqrt{(a / 3 v)} x^{-2 / 3} y_{0}(x)$ the translation group associated with the primary solution (7.1) in its most general form. We see again that for a suitable choice $\eta_{0}(x)$, i.e. of the displacement function $y_{0}(x)$, any desired stretching velocity distribution $\tilde{U}_{w}(x)$ can be prepared from the initial distribution $U_{w}(x)$. According to (5.11) in the present case a suitable $\eta_{0}(x)$ is given by

$$
\eta_{0}(x)=\sqrt{2} \operatorname{arctanh} \sqrt{1-\frac{\tilde{U}_{w}}{U_{w}}}=\frac{\sqrt{2}}{2} \ln \left(\frac{\sqrt{U_{w}}+\sqrt{\tilde{U}_{w}-U_{w}}}{\sqrt{U_{w}}-\sqrt{\tilde{U}_{w}-U_{w}}}\right) .
$$

Accordingly, the velocity field of the generalized Bickley solution induced by an arbitrary stretching velocity distribution $\tilde{U}_{w}(x)$ is

$$
\left.\begin{array}{rl}
\tilde{u}(x, y)= & U_{w} \operatorname{sech}^{2}\left(\frac{\eta}{\sqrt{2}}+\operatorname{arctanh} \sqrt{1-\frac{\tilde{U}_{w}}{U_{w}}}\right), \\
\tilde{v}(x, y)= & -\sqrt{\frac{v U_{w}}{3 x}}\left\{\sqrt{2} \tanh \left(\frac{\eta}{\sqrt{2}}+\operatorname{arctanh} \sqrt{1-\frac{\tilde{U}_{w}}{U_{w}}}\right)\right. \\
& \left.-\left(2 \eta-3 x \eta_{0}^{\prime}\right) \operatorname{sech}^{2}\left(\frac{\eta}{\sqrt{2}}+\operatorname{arctanh} \sqrt{1-\frac{\tilde{U}_{w}}{U_{w}}}\right)\right\} .
\end{array}\right\}
$$

The corresponding transpiration velocity distribution is given by

$$
\tilde{V}_{w}(x)=-\sqrt{\frac{v U_{w}}{3 x}}\left[\sqrt{2\left(1-\frac{\tilde{U}_{w}}{U_{w}}\right)}+3 x \eta_{0}^{\prime} \frac{\tilde{U}_{w}}{U_{w}}\right]=-\sqrt{\frac{v}{6 x}} \frac{2 U_{w}-3 \tilde{U}_{w}-3 x \tilde{U}_{w}^{\prime}}{\sqrt{U_{w}-\tilde{U}_{w}}} .
$$

The self-similarity gets broken also in this case, unless $\tilde{U}_{w}(x)$ is proportional to $U_{w}(x)$, i.e. $\eta_{0}(x)=$ constant. 


\subsection{The case $\eta_{0}(x)=$ constant $\equiv \eta_{0}$}

Taking into account the primary solution (7.1) for $f(\eta)$, the parameters $\tilde{a}, \tilde{f}_{w}$ and $\tilde{f}_{\infty}$ of the transformed solution obtained by a translation with $\eta_{0}(x)=$ constant, as well as the corresponding wall functions (5.15) become

$$
\begin{gathered}
\tilde{a}=a \operatorname{sech}^{2} \frac{\eta_{0}}{\sqrt{2}}, \quad \tilde{f}_{w}=\sqrt{2} \sinh \frac{\eta_{0}}{\sqrt{2}}, \quad \tilde{f}_{\infty}=\sqrt{2} \cosh \frac{\eta_{0}}{\sqrt{2}}, \\
\tilde{U}_{w}(x)=\tilde{a} x^{-1 / 3}, \quad \tilde{V}_{w}(x)=-\sqrt{\frac{v \tilde{a}}{3}} x^{-2 / 3} \tilde{f}_{w} .
\end{gathered}
$$

The latter two (7.7) give the value of the transformed entrainment velocity $\tilde{f}_{\infty}$ in terms of the transformed transpiration parameter $\tilde{f}_{w}$ as

$$
\tilde{f}_{\infty}=\sqrt{\tilde{f}_{w}^{2}+2} .
$$

In this way,

$$
\eta_{0}=\sqrt{2} \operatorname{arctanh} \frac{\tilde{f}_{w}}{\tilde{f}_{\infty}}=\frac{\sqrt{2}}{2} \ln \left(\frac{\sqrt{\tilde{f}_{w}^{2}+2}+\tilde{f}_{w}}{\sqrt{\tilde{f}_{w}^{2}+2}-\tilde{f}_{w}}\right), \quad \tilde{a}=\frac{2 a}{2+\tilde{f}_{w}^{2}},
$$

and thus (5.19) yields for the transformed solution the expressions

$$
\left.\begin{array}{l}
\tilde{u}(x, y)=\tilde{U}_{w}(x) \tilde{f}^{\prime}(\tilde{\eta}), \\
\tilde{v}(x, y)=-\sqrt{\frac{v \tilde{a}}{3}} x^{-2 / 3}\left[\tilde{f}(\tilde{\eta})-2 \tilde{\eta} \tilde{f}^{\prime}(\tilde{\eta})\right],
\end{array}\right\}
$$

where, according to (5.14), (5.18), (6.11) and (7.10)

$$
\left.\begin{array}{l}
\tilde{f}(\tilde{\eta})=\tilde{f}_{\infty} \tanh \left(\frac{\tilde{f}_{\infty}}{2} \tilde{\eta}+\operatorname{arctanh} \frac{\tilde{f}_{w}}{\tilde{f}_{\infty}}\right), \quad \tilde{\eta}=\sqrt{(\tilde{a} / 3 v)} x^{-2 / 3} y, \\
\tilde{f}^{\prime}(\tilde{\eta})=\frac{\tilde{f}_{\infty}^{2}}{2} \operatorname{sech}^{2}\left(\frac{\tilde{f}_{\infty}}{2} \tilde{\eta}+\operatorname{arctanh} \frac{\tilde{f}_{w}}{\tilde{f}_{\infty}}\right) .
\end{array}\right\}
$$

In (7.9), (7.11) and (7.12) we recover the exact solution obtained by Magyari \& Keller (2000) by a direct extension of the primary solution (7.1) to the case of permeable surfaces with transpiration velocity distribution given by the second (7.8) (see also Magyari \& Keller 2001).

$$
\text { 7.3. The case } y_{0}(x)=\text { constant } \equiv y_{0}
$$

In contrast to the case $m=1$ where the displacements $\eta_{0}(x)$ and $y_{0}(x)$ can simultaneously be reduced to a constant, for $m \neq 1$ the constant displacements $y_{0}$ of the transverse coordinate $y$ are associated according to (5.8) with variable displacements $\eta_{0}(x)$ of the similarity independent variable $\eta$. Nevertheless, the transformed velocity field (5.7) simplifies also in the case $y_{0}(x)=$ constant considerably, going over in (5.20). In the present case of the Bickley solution, (5.20) become

$$
\left.\begin{array}{l}
\tilde{u}(x, y)=a x^{-1 / 3} \operatorname{sech}^{2}\left(\frac{\eta+\eta_{0}}{\sqrt{2}}\right), \quad \eta_{0}(x)=\sqrt{\frac{a}{3 v}} y_{0} x^{-2 / 3}, \\
\tilde{v}(x, y)=-\sqrt{\frac{v a}{3}} x^{-2 / 3}\left\{\sqrt{2} \tanh \left(\frac{\eta+\eta_{0}}{\sqrt{2}}\right)-2\left(\eta+\eta_{0}\right) \operatorname{sech}^{2}\left(\frac{\eta+\eta_{0}}{\sqrt{2}}\right)\right\} .
\end{array}\right\}
$$




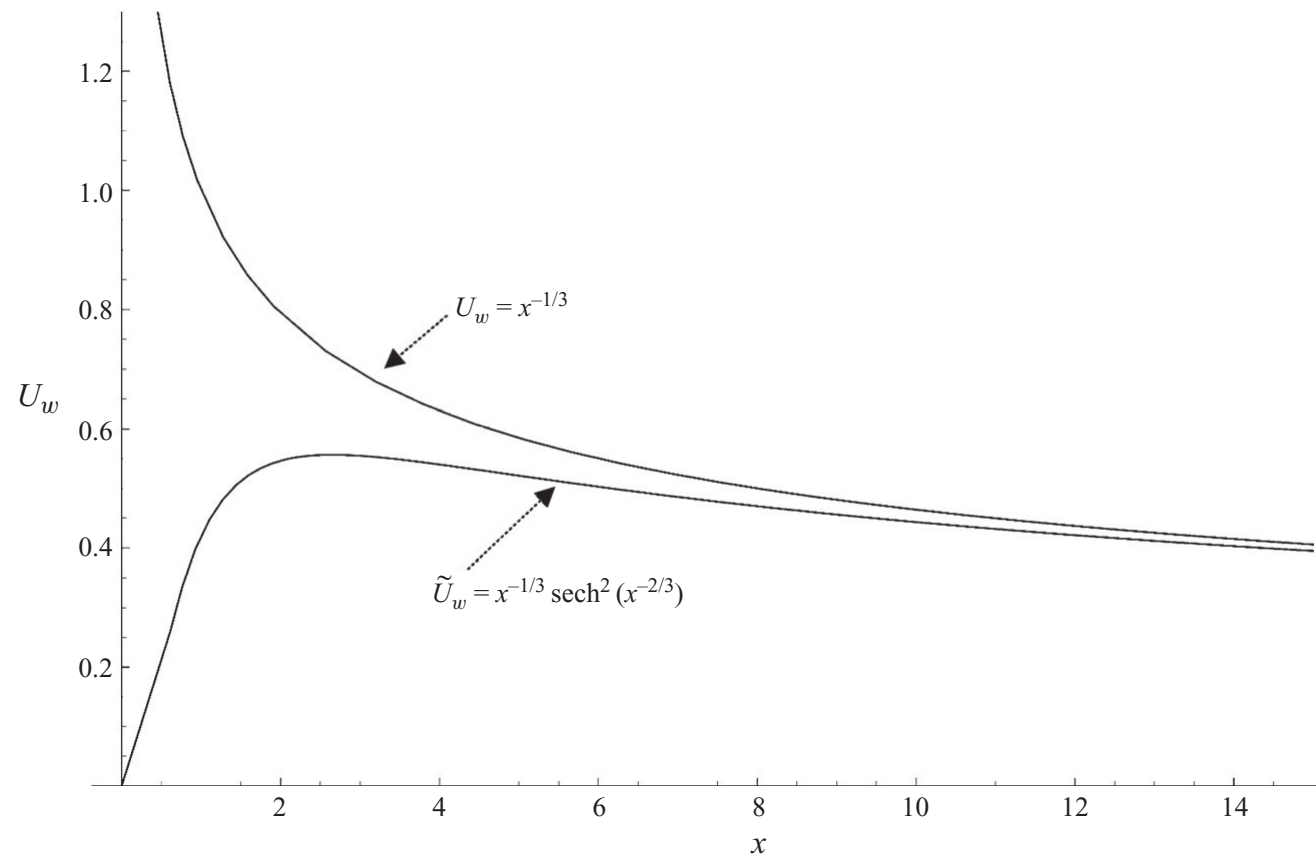

FIGURE 1. Shapes of the stretching velocity distributions $U_{w}$ and $\tilde{U}_{w}$ corresponding to the Bickley solution (7.1) and its transformed counterpart (7.14) obtained by a constant translation $y_{0}$ of the transverse coordinate $y$ (the velocities have been expressed in units of $\left(6 a^{3} v / y_{0}^{2}\right)^{1 / 4}$ and the coordinate $x$ in units of $\left.\left(a y_{0}^{2} / 6 v\right)^{3 / 4}\right)$.

The stretching and transpiration velocity distributions which induce this new solution of our boundary value problem are

$$
\left.\begin{array}{rl}
\tilde{U}_{w}(x)= & a x^{-1 / 3} \operatorname{sech}^{2}\left(\sqrt{\frac{a}{6 v}} y_{0} x^{-2 / 3}\right), \\
\tilde{V}_{w}(x)= & -\sqrt{\frac{v a}{3}} x^{-2 / 3}\left\{\sqrt{2} \tanh \left(\sqrt{\frac{a}{6 v}} y_{0} x^{-2 / 3}\right)\right. \\
& \left.-2 \sqrt{\frac{a}{6 v}} y_{0} x^{-2 / 3} \operatorname{sech}^{2}\left(\sqrt{\frac{a}{6 v}} y_{0} x^{-2 / 3}\right)\right\} \cdot
\end{array}\right\}
$$

The self-similarity of the primary solution (7.1) gets in the transformed solution (7.13) broken. It is also seen that for small values of $x$ the transformed stretching velocity $\tilde{U}_{w}(x)$ given by the first (7.14) deviates substantially from the initial one $U_{w}(x)=a x^{-1 / 3}$, but approaches it far downstream. These features are illustrated in figure 1 where the two stretching velocities $U_{w}$ and $\tilde{U}_{w}$ have been plotted as functions $x$.

\section{Translation group of the universal backward boundary-layer solution}

It is known that the boundary value problem (5.3)-(5.4) is formally equivalent (up to scaling factors of $\eta$ and $f$ ) to the boundary value problem arising in the ChengMinkowycz model (Cheng \& Minkowycz 1977) of the free convection boundary-layer flow past a heated vertical surface in a fluid saturated porous medium (see also Pop \& Ingham 2001). In the latter context, it has been shown by Magyari \& Keller (2004) 
that a universal backward boundary-layer solution exists which holds for any power law exponent $m$. In the present case of the boundary value problem (5.3)-(5.4) with

$$
U_{w}(x)=a x^{m}, \quad a<0, \quad s=-\operatorname{sgn}(m+1),
$$

this universal solution has the form

$$
f(\eta)=-\frac{f_{w}^{2}}{\eta-f_{w}},
$$

where

$$
f_{w}=-\sqrt{3|m+1|}<0 \quad(m \neq-1) .
$$

We mention that for $m=1$, (8.2) coincides with the solution reported recently by Fang \& Zhang (2010).

The velocity field (5.2) corresponding to the dimensionless stream function (8.2) is

$$
\left.\begin{array}{l}
u(x, y)=\frac{f_{w}^{2} U_{w}(x)}{\left(\eta-f_{w}\right)^{2}}, \quad \eta=\sqrt{\frac{|a(m+1)|}{2 v}} x \frac{m-1}{2} y, \\
v(x, y)=3 \sqrt{\frac{v\left|(m+1) U_{w}\right|}{2 x}} \frac{(m+1) f_{w}-2 \eta}{\left(\eta-f_{w}\right)^{2}},
\end{array}\right\}
$$

and it is associated with the wall functions

$$
U_{w}(x)=-|a| x^{m}, \quad V_{w}(x)=-(m+1) \sqrt{\frac{3 v\left|U_{w}(x)\right|}{2 x}} .
$$

The second (8.5) shows that the existence of the universal solution (8.4) requires a lateral suction of the fluid when $m>-1$ and a lateral injection when $m<-1$. Thus, the solution (8.4) is not a primary solution in the sense defined in this paper. Nevertheless, by translations (3.1) of the transverse coordinate it can generate new solutions corresponding to any desired stretching velocity $\tilde{U}_{w}(x)$. Indeed, in this case (5.11) yields

$$
\eta_{0}(x)=f_{w}\left(1-\sqrt{\frac{U_{w}(x)}{\tilde{U}_{w}(x)}}\right) .
$$

Thus, according to (5.9) and (8.2) the transformed counterpart of the velocity field (8.4) is

$$
\left.\begin{array}{l}
\tilde{u}(x, y)=\frac{f_{w}^{2} U_{w}(x)}{\left(\eta-f_{w} \sqrt{U_{w}(x) / \tilde{U}_{w}(x)}\right)^{2}}, \quad \eta=\sqrt{\frac{\left|(m+1) U_{w}(x)\right|}{2 v x}} y, \\
v(x, y)=3 \sqrt{\frac{v\left|(m+1) U_{w}\right|}{2 x}} \frac{(m+1) f_{w} \sqrt{\frac{U_{w}}{\tilde{U}_{w}}}-2 \eta-f_{w} x \sqrt{\frac{\tilde{U}_{w}}{U_{w}}} \frac{d}{d x}\left(\frac{U_{w}}{\tilde{U}_{w}}\right)}{\left(\eta-f_{w} \sqrt{U_{w} / \tilde{U}_{w}}\right)^{2}} .
\end{array}\right\}
$$

The transpiration velocity distribution corresponding to this transformed velocity field is

$$
\tilde{V}_{w}(x)=-\sqrt{\frac{3 v\left|U_{w}\right|}{2 x}}\left(\frac{\tilde{U}_{w}}{U_{w}}\right)^{1 / 2}\left[(m+1)-x \frac{\tilde{U}_{w}}{U_{w}} \frac{\mathrm{d}}{\mathrm{d} x}\left(\frac{U_{w}}{\tilde{U}_{w}}\right)\right] .
$$

The self-similarity gets broken also in this case, unless $\tilde{U}_{w}(x)$ is proportional to $U_{w}(x)$, i.e. $\eta_{0}(x)=$ constant. 


\section{Summary and conclusions}

The boundary value problem which describes the steady plane boundary-layer flows induced by continuous moving surfaces has been revisited in the present paper. The main results of this investigation can be summarized as follows.

(a) The governing balance equations as well as the asymptotic condition $u(x, \infty)=0$ at the outer edge of the boundary layer are invariant under arbitrary translations $y \rightarrow y+y_{0}(x)$ of the transverse coordinate $y$. The prescribed stretching velocity $u(x, 0) \equiv U_{w}(x)$ and the transpiration velocity $v(x, 0) \equiv V_{w}(x)$ distributions, however, change under this transformation as $U_{w}(x) \rightarrow \tilde{U}_{w}(x)=u\left[x, y_{0}(x)\right]$ and $V_{w}(x) \rightarrow \tilde{V}_{w}(x)=v\left[x, y_{0}(x)\right]-y_{0}^{\prime}(x) u\left[x, y_{0}(x)\right]$, respectively.

(b) As a consequence, from a given solution $\{u(x, y), v(x, y)\}$ of the boundary value problem, by the translations $y \rightarrow y+y_{0}(x)$ infinitely many new solutions $\{\tilde{u}(x, y), \tilde{v}(x, y)\}$ can be generated which form a continuous group, the translation group of the given solution.

(c) The elements of the translation group describe boundary-layer flows induced by surfaces stretching under the transformed wall conditions $\left\{\tilde{U}_{w}(x), \tilde{V}_{w}(x)\right\}$. When the definition equation $u\left[x, y_{0}(x)\right]=\tilde{U}_{w}(x)$ of $\tilde{U}_{w}(x)$ can be inverted and solved for $y_{0}(x)$ explicitly, then $y_{0}(x)=u^{-1}\left(x, \tilde{U}_{w}\right)$ yields that special translation of transverse coordinate $y$ which generates from the primary solution $\{u(x, y), v(x, y)\}$ precisely that new solution $\{\tilde{u}(x, y), \tilde{v}(x, y)\}$ which correspond to a desired stretching velocity distribution $\tilde{U}_{w}(x)$.

(d) It also turns out that several exact solutions discovered in the past are not basically new solutions, but translated counterparts of some formerly reported solutions.

The features described above emphasize once more the paramount importqance of the transpiration velocity distribution in the boundary-layer control. Thus, we can imagine that the freedom conferred by the translation group possesses the capability to become important for the fabrication of some new two-dimensional porous materials. It is also worth mentioning here that the results of the present paper can directly be extended to the cases when the momentum equation has the form $u u_{x}+v u_{y}=v u_{y y}+F(u)$, where $F(u)$ denotes some function of the mainstream velocity $u$. This applies, for example, to the case of hydromegnetic flows of electrically conducting fluids driven by a continuous stretching surface in a constant transverse magnetic field. In this case $F(u)$ coincides with the Hartmann-drag term, which is proportional to $-u$.

\section{REFERENCES}

ACROYD, J. A. D. 1967 On the laminar compressible boundary layer with stationary origin on a moving flat wall. J. Fluid Mech. 63, 871-888.

AfZAL, N. \& VARShney, I. S. 1980 The cooling of a low heat resistance stretching sheet moving through a fluid. Wärme-Stoffübertrag. 14, 289-293.

Ali, M. E. 1995 On thermal boundary layer on a power law stretched surface with suction and injection. Intl J. Heat Fluid Flow 16, 280-290.

Altan, T., OH, S. \& Gegel, H. 1979 Metal Forming Fundamentals and Applications. American Society of Metals.

BANKs, W. H. H. 1983 Similarity solutions of the boundary layer equations for a stretching wall. J. Méc. Theor. Appl. 2, 375-392.

Bickley, W. G. 1937. The plane jet. Phil. Mag. 23, 727-731.

Chen, C. K. \& Char, M. 1988 Heat transfer of a continuous stretching surface with suction and blowing. J. Math. Anal. Appl. 135, 248-250. 
Cheng, P. \& Minkowycz, W. J. 1977 Free convection about a vertical flat plate embedded in a porous medium with application to heat transfer from a dike. J. Geophys. Res. 82, 2040-2044.

Crane, L. E. 1970 Flow past a stretching plane. J. Appl. Math. Phys. (ZAMP) 21, 645-647.

FANG, T \& ZHANG, J. 2010 Thermal boundary layers over a shrinking sheet: an analytical solution. Acta Mech. 209, 325-343.

FISHER, E. G. 1976 Extrusion of Plastics. Wiley.

Fox, V. G., Erickson, L. E. \& FAN, L. T. 1968 Methods for solving the boundary layer equations for moving continuous flat surfaces with suction and injection. AIChE J. 14, 726-736.

Goldstein, S. 1939 A note on the boundary layer equations. Proc. Camb. Phil. Soc. 35, 338-340.

GoldsteIn, S. 1965 On backward boundary layers and flow in converging passages. J. Fluid Mech. 21, 33-45.

GrubKa, L. J. \& BobBa, K. M. 1985 Heat transfer characteristics of a continuous stretching surface with variable temperature. J. Heat Transfer 107, 248-250.

Gupta, P. S. \& Gupta, A. S. 1977 Heat and mass transfer on a stretching sheet with suction or blowing. Can. J. Chem. Engng 55, 744-746.

INGHAM, D. B. \& BRown, S. N. 1986 Flow past a suddenly heated vertical plate in a porous medium. Proc. R. Soc. Lond. A 403, 51-80.

Kuiken, H. K. 1981 On boundary layer in fluid mechanics that decay algebraically along stretches of wall that are not vanishingly small. IMA J. Appl. Math. 27, 387-405.

LiaO, S. J. 2006 Series solutions of unsteady boundary-layer flows over a stretching flat plate. Stud. Appl. Math. 117, 239-263.

LiaO, S. J. \& MagYaRI, E. 2006 Exponentially decaying boundary layers as limiting cases of families of algebraically decaying ones. J. Appl. Math. Phys. (ZAMP) 57, 777-792.

MagYaRI, E. 2008 The Entrainment Theorem for wall driven boundary layer flows. Acta Mec. 201, $119-129$.

Magyari, E., Ali, M. E. \& Keller, B. 2001 Heat and mass transfer characteristics of the selfsimilar boundary-layer flows induced by continuous surfaces stretched with rapidly decreasing velocities. Heat Mass Transfer 38, 65-74.

MagYaRi, E. \& Keller, B. 1999 Heat and mass transfer in the boundary layers on an exponentially stretching continuous surface. J. Phys. D: Appl. Phys. 32, 2876-2881.

MagYari, E. \& Keller, B. 2000 Exact solutions for self-similar boundary-layer flows induced by permeable stretching walls. Eur. J. Mech. B/Fluids 19, 109-122.

Magyari, E. \& Keller, B. 2001 The free jets as boundary layer flows induced by continuous stretching surfaces. Heat Mass Transfer 38, 111-114.

MagYARI, E. \& KelLeR, B. 2004 Backward free convection boundary layers in porous media. Transp. Porous Media 55, 285-300.

MagYaRi, E. \& Keller, B. 2005 A direct method to calculate the heat transfer coefficient of steady similar boundary layer flows induced by continuous moving surfaces. Intl J. Therm. Sci. 44, 245-254.

Magyari, E., Pop, I. \& Keller, B. $2002 a$ The missing self-similar free convection boundary layer flow over a vertical surface in a porous medium. Transp. Porous Media 46, 91-102.

Magyari, E., Pop, I. \& Keller, B. 2002b The missing similarity boundary-layer flow over a moving plane surface. J. Appl. Math. Phys. (ZAMP) 53, 782-793.

Magyari, E., Pop, I. \& Keller, B. 2003 New analytical solutions of a well known boundary value problem in fluid mechanics. Fluid Dyn. Res. 33, 351-355.

Magyari, E. \& ReEs, D. A. S. 2006 Effect of viscous dissipation on the Darcy free convection flow over a vertical plate with exponential temperature distribution in a porous medium. Fluid Dyn. Res. 38, 405-429.

Magyari, E. \& Rees, D. A. S. 2008 The Entrainment Theorem for the Darcy free convection over a permeable vertical plate. Intl J. Therm. Sci. 47, 1123-1130.

Merkin, J. H. 1984 A note on the solution of a differential equation arising in boundary-layer theory. J. Engng Math. 18, 31-36.

Pop, I. \& Ingham, D. B. 2001 Convective Heat Transfer: Mathematical and Computational Modelling of Viscous Fluids and Porous Media. Pergamon.

SAKIADIS, B. C. 1961. Boundary-layer behavior on continuous solid surfaces. AIChE J. 7, 26-28. See also pp. 221-225 and 467-472. 
Tadmor, Z. \& Klein, I. 1970 Engineering Principles of Plasticating Extrusion. Polymer Science and Engineering Series. Van Norstrand Reinhold.

Tsou, F. K., Sparrow, E. M. \& Goldstein, R. J. 1967 Flow and heat transfer in the boundary layer on a continuous moving surface. Intl J. Heat Mass Transfer 10, 219-235.

VleggaAR, J. 1977 Laminar boundary layer behavior on continuous, accelerating surfaces. Chem. Engng Sci. 32, 1517-1525.

Weidman, P. D. \& MagYaRi, E. 2010 Generalized Crane flows induced by continuous surfaces stretching with arbitrary velocities. Acta Mech. 209, 353-362. 